\title{
A Throughput and Delay Model for IEEE 802.11e EDCA Under Non Saturation
}

\author{
PABLO SERRANO, ALBERT BANCHS and ARTURO AZCORRA \\ Universidad Carlos III de Madrid, Madrid, Spain \\ E-mails: pablo@it.uc3m.es, banchs@it.uc3m.es,azcorra@it.uc3m.es
}

\begin{abstract}
In this paper, we present a model to analyze the throughput and delay performance of the EDCA mechanism under non saturation conditions. The main strength of our model is that it can be used to analyze generic source models, as it neither makes any assumption on the source's arrival process nor requires all packets be of the same length. Simulation results confirm the accuracy of our model under a variety of realistic source models, including $(i)$ typical arrival processes for voice, video, and data traffic, and ( $i i)$ packet length distributions derived from measurements.
\end{abstract}

Keywords: WLAN, 802.11e, EDCA, delay analysis, throughput analysis, non saturation

\section{Introduction}

In recent years, much interest has been devoted to the design of wireless local area networks (WLAN's) with Quality of Service (QoS) support. The Enhancements Task Group (TGe) was formed under the IEEE 802.11 Working Group to recommend an international WLAN standard with QoS support. This standard is called 802.11e and has been recently approved [1]. The standard defines two different access mechanisms: the Enhanced Distributed Channel Access (EDCA) and the HCF Controlled Channel Access (HCCA). Our focus here is on the former.

To date, there has been a remarkable amount of work to evaluate the throughput performance of 802.11e EDCA analytically. However, most of the existing analyses [2-8] are based on the unrealistic assumption that all stations always have packets ready for transmission. This is commonly referred to as saturation conditions. In this paper, we propose a novel model for EDCA that, unlike these analyses, does not assume saturation conditions but works for finite loads.

Although some previous analyses of non saturated WLANs have been proposed in the literature [9-13], these are typically valid only for Poisson arrivals and restricted to fixed length packets. In contrast to these previous papers, our analysis here does not make any assumption on the arrival process and allows for variable packet lengths. Indeed, our simulation results, which are based on different arrival models and variable packet lengths, show the validity and accuracy of our analysis under such conditions.

The rest of the paper is structured as follows. In Section 2, we briefly summarize the 802.11e EDCA mechanism. In Section 3, an analysis of the throughput and delay performance of EDCA under non saturation traffic conditions is presented. The accuracy of the 
presented analysis is validated by the simulations results presented in Section 4. Finally, the paper closes with some final remarks in Section 5.

\section{802.11e EDCA}

This section briefly summarizes the EDCA mechanism as defined in the 802.11e standard. EDCA controls the access to the wireless channel on the basis of the Channel Access Functions (CAF's). To transmit its frames, each CAF executes an independent backoff process which is regulated by a number of configurable parameters. For the configuration of these parameters, the standard groups the CAF's by Access Categories (ACs) and assigns the same configuration to all the CAF's of an AC. In this paper, we assume for simplicity that each station runs only one CAF and use indistinctly the terms CAF and station. ${ }^{1}$

A station of an Access Category $i\left(\mathrm{AC}_{i}\right)$ with a new frame to transmit monitors the channel activity. If the channel is idle for a period of time equal to the arbitration interframe space parameter of this $\mathrm{AC}\left(\mathrm{AIFS}_{i}\right)$, the station transmits. Otherwise, if the channel is sensed busy (either immediately or during the $\mathrm{AIFS}_{i}$ period), the station continues to monitor the channel until it is measured idle for an AIFS $_{i}$ time, and, at this point, the backoff process starts. The $\mathrm{AIFS}_{i}$ takes a value of the form DIFS $+n \sigma$, where DIFS and $\sigma$ are constants dependent on the physical layer and $n$ is a nonnegative integer. ${ }^{2}$

Upon starting the backoff process, the station computes a random value uniformly distributed in the range $\left(0, \mathrm{CW}_{i}-1\right)$, and initializes its backoff time counter with this value. The $\mathrm{CW}_{i}$-value is called the contention window, and depends on the number of transmissions failed for the frame. At the first transmission attempt, $\mathrm{CW}_{i}$ is set equal to the minimum contention window parameter $\left(\mathrm{CW}_{i}^{\mathrm{min}}\right)$. As long as the channel is sensed idle the backoff time counter is decremented once every time interval $\sigma$. When a transmission is detected on the channel, the backoff time counter is "frozen", and reactivated again after the channel is sensed idle for a certain period (equal to $\mathrm{AIFS}_{i}$ if the transmission is received with a correct CRC, and equal to EIFS - DIFS + AIFS $_{i}$ otherwise).

As soon as the backoff time counter reaches zero, the station transmits its frame in the next slot time. A collision occurs when two or more stations start transmission simultaneously. An acknowledgement (Ack) frame is used to notify the transmitting station that the frame has been successfully received. If the Ack is not received within a given timeout, the station assumes that the frame was not received and reschedules the transmission by reentering the backoff process. After each unsuccessful transmission $\mathrm{CW}_{i}$ is doubled, up to a maximum value given by the $\mathrm{CW}_{i}^{\mathrm{max}}$ parameter. If the number of failed attempts reaches a predetermined retry limit $R$, the frame is discarded. Once the backoff process is completed (either successfully or unsuccessfully), $\mathrm{CW}_{i}$ is set again to $\mathrm{CW}_{i}^{\mathrm{min}}$.

When the station gains access to the channel, it is allowed to retain the right to access it for a duration equal to the transmission opportunity limit parameter (TXOP_limit ${ }_{i}$ ). If this parameter is set to zero, a station is allowed to transmit only one packet upon accessing the

\footnotetext{
1 Note that, following the lines of [7], the analysis here could easily be extended to the case of multiple CAF's per station.

2 According to the IEEE 802.11e standard terminology, AIFS $_{i}=\mathrm{SIFS}+n \sigma$, where DIFS $=$ SIFS $+2 \sigma$ and $n \geq 2$. Without loss of generality, in this paper we use the simplified notation $\operatorname{AIFS}_{i}=\operatorname{DIFS}+n \sigma$, with $n \geq 0$.
} 
channel. In the rest of this paper, we assume this setting for the TXOP_limit ${ }_{i}$ parameter, and concentrate on the analysis of the other three parameters $\left(\mathrm{CW}_{i}^{\min }, \mathrm{CW}_{i}^{\max }\right.$ and $\left.\mathrm{AIFS}_{i}\right){ }^{3}$

\section{Throughput and Delay Analysis}

In this section, we consider a WLAN operating under the EDCA mechanism and analyze the throughput and the delay of each AC in the WLAN. The input parameters to our analysis are:

- The number of AC's in the WLAN (which we denote by $N$ ).

- The number of stations of each AC (we denote by $n_{i}$ the number of stations of $\mathrm{AC} i$ ).

- The average sending rate of the stations of each AC (denoted by $\rho_{i}$ ).

- The configuration $\left\{\mathrm{CW}_{i}^{\mathrm{min}}, m_{i}, A_{i}\right\}$ of each AC, where $m_{i}$ is defined such that $\mathrm{CW}_{i}^{\max }=$ $2^{m_{i}} \mathrm{CW}_{i}^{\mathrm{min}}$ and $A_{i}$ such that $\mathrm{AIFS}_{i}=\mathrm{DIFS}+A_{i} \sigma$.

The key approximation upon which we base our analysis is centered on the notion of saturation rate. By the saturation rate of an $\mathrm{AC}$ we understand the rate that the stations of this AC would obtain if they always had a packet ready for transmission. Based on this, our assumptions are: 4

- As long as the average sending rate of the stations of a given AC falls below the AC's saturation rate, we assume that the stations of this AC see all their packets served (i.e., their transmission queue never overflows). We refer to such an $\mathrm{AC}$ as a non saturated AC.

- On the other hand, if the average sending rate of the stations of the AC exceeds the saturation rate, we consider that the stations of this AC always have packets ready for transmission (i.e., their transmission queue never empties). We say that such an AC is saturated.

The key variable upon which we build our analysis is $\tau_{i}$, defined as the probability that a station of AC $i$ transmits upon a backoff counter decrement. In the following, we first analyze separately the $\tau_{i}$ of a saturated $\mathrm{AC}$ and the $\tau_{i}$ of a non saturated $\mathrm{AC}$, respectively. Then, we combine both analyses in order to compute the $\tau_{i}$-values of all the AC's in the WLAN. Finally, based on these values, we calculate the throughput and the delay of each AC.

\subsection{Analysis of a Saturated AC}

Let us start with the case of a saturated AC [8]. With the assumption of [14] that each transmission attempt collides with a constant and independent probability, we can model the behavior of this AC with the same Markov chain as Figure 5 of [14]. Then, the probability that a station

\footnotetext{
${ }^{3}$ Note that the impact of the TXOP_limit ${ }_{i}$ parameter is typically small in realistic scenarios. In fact, for realtime traffic parameters are usually set such that the queue never grows to more than one packet, and therefore this parameter is not used, while for data traffic this parameter is set such that only one packet is transmitted upon accessing the channel, in order to avoid degrading the delay performance of real-time traffic.

${ }^{4}$ Note that, as these assumptions rely on no source property other than the average sending rate, our model can be applied to analyze generic source models. The only restriction imposed on the sources is that they be stationary, as otherwise their average sending rate could change over time. Even in that case, our model could be used to analyze each stationary time interval separately.
} 


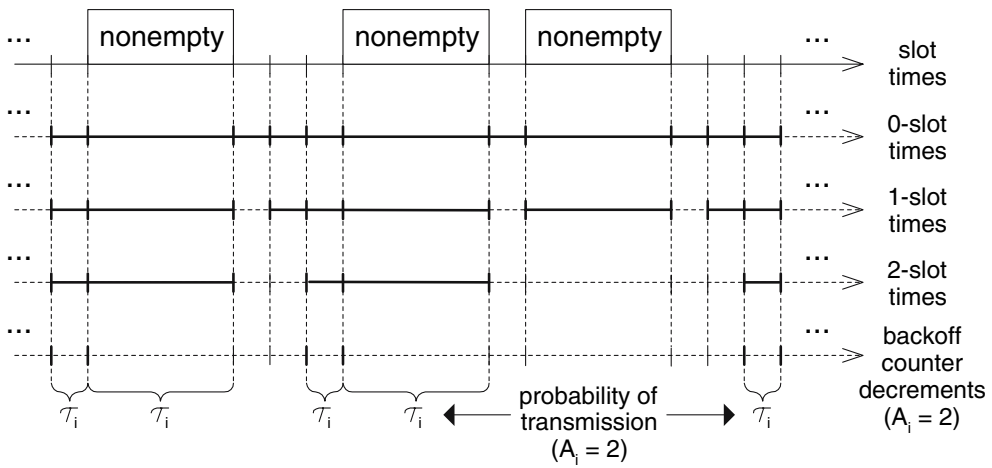

Figure 1. $k$-slot times and probability of transmission (example with $k=2$ ).

of a saturated $\mathrm{AC}$ transmits upon a backoff counter decrement can be computed following the well known result of [14],

$$
\tau_{i}^{\mathrm{sat}}=\frac{2\left(1-2 p_{i}\right)\left(1-p_{i}^{R+1}\right)}{\mathrm{CW}_{i}^{\min }\left(1-\left(2 p_{i}\right)^{m_{i}+1}\right)\left(1-p_{i}\right)+\left(1-2 p_{i}\right)\left(1-p_{i}^{R+1}\right)+\mathrm{CW}_{i}^{\min } 2^{m_{i}} p_{i}^{m_{i}+1}\left(1-2 p_{i}\right)\left(1-p_{i}^{R-m_{i}}\right)},
$$

where $p_{i}$ is the probability that a transmission attempt of a station of AC $i$ collides.

To compute $p_{i}$, we proceed as follows. We start by defining a slot time as the time interval between two consecutive backoff counter decrements of a station with minimal AIFS $_{i}$ (i.e., DIFS). We say that a slot time is nonempty when it contains a collision or a successful transmission and that it is empty otherwise.

We further define a $k$-slot time as a slot time that is preceded by $k$ or more empty slot times. Note that, since a station with $A_{i}=k$ starts decrementing its backoff counter only after $k$ empty slot times following a nonempty slot time, we have that the backoff counter decrements of this station coincide with the boundaries of the $k$-slot times. Therefore, a station of $\mathrm{AC} i$, with $A_{i}=k$, transmits in a $k$-slot time with probability $\tau_{i}$, and does not transmit in any other slot time (see Figure 1).

Based on the above definitions, we compute $p_{i}$ as a function of the probability of an empty $k$-slot time (denoted by $p\left(e_{k}\right)$ ) as follows. A $k$-slot time is empty as long as $(i)$ the considered station does not transmit, and ( $i i)$ no other station transmits. The latter can be expressed as a function of $p_{i}$ by noting that the probability of a collision corresponds to the case when some other station transmits. Thus,

$$
p\left(e_{k}\right)=\left(1-\tau_{i}\right)\left(1-p_{i}\right)
$$

which yields

$$
p_{i}=1-\frac{p\left(e_{k}\right)}{1-\tau_{i}} .
$$

Now let us focus on the probability that a given $k$-slot time is empty. If the previous $k$-slot time was nonempty, in this $k$-slot time only the AC's with $A_{i} \leq k$ may transmit. If the previous $k$-slot time was empty, the given $k$-slot time is preceded by $k+1$ or more empty slot times, which is exactly the definition of $(k+1)$-slot time, and therefore such a $k$-slot time is empty 


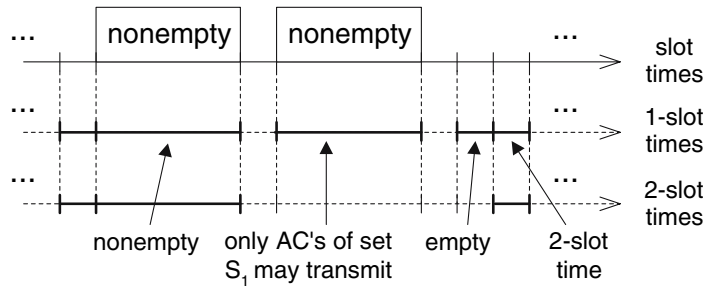

Figure 2. Probability of an empty $k$-slot time (example with $k=1$ ).

with probability $p\left(e_{k+1}\right)$. Applying this reasoning (see Figure 2 ), $p\left(e_{k}\right)$ can be written as

$$
p\left(e_{k}\right)=\left(1-p\left(e_{k}\right)\right) \prod_{j \in A C_{k}}\left(1-\tau_{j}\right)^{n_{j}}+p\left(e_{k}\right) p\left(e_{k+1}\right),
$$

where $A C_{k}$ is the set of AC's with $A_{i} \leq k$.

Let $\Delta$ be the largest $A_{i}$ in the WLAN. As (with this definition of $\Delta$ ) in a $\Delta$-slot time all stations may transmit, the following equation holds

$$
p\left(e_{\Delta}\right)=\prod_{j \in A C_{\Delta}}\left(1-\tau_{j}\right)^{n_{j}}
$$

Starting from $\tau_{i} \forall i$, with Eq. (5) we can compute $p\left(e_{\Delta}\right)$. Then, with Eq. (4) for $k=\Delta-1$, we can compute $p\left(e_{\Delta-1}\right)$. Applying this recursively, we can compute $p\left(e_{k}\right) \forall k$. Then, $p_{i}$ can be computed via Eq. (3) and, finally, $\tau_{i}^{\text {sat }}$ can be obtained from Eq. (1). As result, we can express the $\tau_{i}$ of a saturated $\mathrm{AC}, \tau_{i}^{\text {sat }}$, as a function of all the $\tau_{i}$ 's. This terminates the analysis for this case.

\subsection{Analysis of a Non Saturated AC}

We next focus on the analysis of a non saturated AC. According to our previous assumption, a station of a non saturated AC sees all the traffic it sends served, either because their packets are transmitted successfully or because they are discarded when reaching the retry limit. Hence, the following equation holds,

$$
\rho_{i}\left(1-p_{i}^{R+1}\right)=r_{i}
$$

where $r_{i}$ is the throughput experienced by a station of AC $i$ (i.e., the successful transmission rate), $\rho_{i}$ is its average sending rate and $p_{i}^{R+1}$ corresponds to the probability that a packet of this station is discarded upon reaching the retry limit.

The throughput $r_{i}$ is computed as the average payload information transmitted in a slot time divided by the average duration of a slot time:

$$
r_{i}=\frac{p\left(s_{i}\right) l_{i}}{p(s) T_{s}+p(c) T_{c}+p(e) \sigma},
$$

where $l_{i}$ is the average packet length of a station of AC $i, p\left(s_{i}\right)$ is the probability that a randomly chosen slot time contains a successful transmission of a station of $\mathrm{AC} i, p(s), p(c)$, and $p(e)$ are the probabilities that a slot time contains a successful transmission, a collision or is empty, respectively, and $T_{\mathrm{s}}, T_{\mathrm{c}}$, and $\sigma$ are the average slot time durations in each case. 
The probability $p(e)$ is, by definition, $p\left(e_{0}\right)$, as all slot times are 0 -slot times. This has already been computed in Section 3.1.

Let us define $p_{k}$ as the probability that a slot time is a $k$-slot time. Since a slot time is a $k$-slot time if and only if the previous slot time is a $(k-1)$-slot time and it is empty, this probability can be expressed as

$$
p_{k}=p_{k-1} p\left(e_{k-1}\right)
$$

Starting from $p_{0}=1$ (which holds by definition), it follows

$$
p_{k}=\prod_{j=0}^{k-1} p\left(e_{j}\right)
$$

The probability that a random slot time contains a success of a given station of AC $i$ can be computed as

$$
p\left(s_{i}\right)=\sum_{k=A_{i}}^{\Delta} p\left(A C_{k}\right) p\left(s_{i} \mid A C_{k}\right),
$$

where $p\left(A C_{k}\right)$ is the probability that a randomly chosen slot time is allowed for transmission to only the AC's of set $A C_{k}$, and $p\left(s_{i} \mid A C_{k}\right)$ is the probability that a slot time in which only the AC's of set $A C_{k}$ may transmit contains a success of a given station of $\mathrm{AC} i$.

A slot time is allowed for transmission to only the AC's of set $A C_{k}$ (with $k<\Delta$ ) if the slot time is a $k$-slot time but not a $(k+1)$-slot time. ${ }^{5}$ For $k=\Delta$, we have that in a $\Delta$-slot time all AC's are allowed to transmit. Thus,

$$
p\left(A C_{k}\right)= \begin{cases}p_{k}-p_{k+1}, & k<\Delta, \\ p_{\Delta}, & k=\Delta .\end{cases}
$$

The probability $p\left(s_{i} \mid A C_{k}\right)$ corresponds to the case when the considered station transmits and no other station of set $A C_{k}$ does:

$$
p\left(s_{i} \mid A C_{k}\right)=\tau_{i}\left(1-\tau_{i}\right)^{n_{i}-1} \prod_{j \in A C_{k} \backslash i}\left(1-\tau_{j}\right)^{n_{j}} .
$$

The probability that a slot time contains a success can be computed as the sum of the individual success probabilities:

$$
p(s)=\sum_{i \in A C_{\Delta}} n_{i} p\left(s_{i}\right)
$$

The average duration of a success can be computed according to

$$
T_{\mathrm{s}}=\sum_{i \in A C_{\Delta}} \frac{n_{i} p\left(s_{i}\right)}{p(s)} T_{\mathrm{s}}^{i}
$$

\footnotetext{
5 Note that a slot time that is a $k$-slot time but not a $(k+1)$-slot time is preceded by exactly $k$ empty slot times, and therefore only the AC's with $A_{i} \leq k$ (i.e., the AC's of set $A C_{k}$ ) may transmit in such a slot time.
} 
where $T_{\mathrm{s}}^{i}$ is the average duration of a success of a station of $\mathrm{AC} i$, which is calculated according to

$$
T_{\mathrm{s}}^{i}=T_{\mathrm{PLCP}}+\frac{H+l_{i}}{C}+\mathrm{SIFS}+T_{\mathrm{PLCP}}+\frac{\mathrm{ACK}}{C}+\mathrm{DIFS}
$$

where $T_{\mathrm{PLCP}}$ is the Physical Layer Convergence Protocol preamble and header transmission time, $H$ the MAC overhead (header and Frame Check Sequence), ACK the size of the acknowledgement frame, and $C$ is the channel bit rate.

The probability that a slot time contains a collision can be obtained from

$$
p(c)=1-p(e)-p(s)
$$

In order to compute the average duration of a collision, we note that this is given by the largest packet length involved in the collision. Thus,

$$
T_{\mathrm{c}}=\sum_{l \in L} \frac{p\left(c_{l}\right)}{p(c)} T_{\mathrm{c}}^{l}
$$

where $p\left(c_{l}\right)$ is the probability that a slot time contains a collision in which the length of the longest packet involved is equal to $l, T_{\mathrm{c}}^{l}$ the duration of this collision, and $L$ is the set of all possible packet lengths.

$T_{\mathrm{c}}^{l}$ is computed as

$$
T_{\mathrm{c}}^{l}=T_{\mathrm{PLCP}}+\frac{H+l}{C}+\mathrm{EIFS}
$$

and $p\left(c_{l}\right)$ as

$$
p\left(c_{l}\right)=\sum_{k=0}^{\Delta} p\left(A C_{k}\right) p\left(c_{l} \mid \mathrm{AC}_{k}\right)
$$

where $p\left(c_{l} \mid A C_{k}\right)$ is the probability that, given that only stations of set $\mathrm{AC}_{k}$ may transmit, a slot time contains a collision with the longest packet involved of length $l$.

To obtain $p\left(c_{l} \mid A C_{k}\right)$ we proceed as follows: we sweep along all the stations that may transmit and compute the probability that $(i)$ the considered station transmits a packet of length $l$, (ii) some other station transmits, and ( $i$ ii) no packet longer than $l$ is transmitted. Let us define $S_{k}$ as the set of stations of $\mathrm{AC}_{k}, \tau_{j}$ as the probability that station $j$ transmits and $p\left(t_{j}=l\right)$ as the probability that its transmission length is equal to $l$. Then,

$$
p\left(c_{l} \mid \mathrm{AC}_{k}\right)=\sum_{j \in S_{k}} \tau_{j} p\left(t_{j}=l\right) p(\text { no } t x>l) p(\text { some } t x)
$$

where $p($ no $t x>l)$ is the probability that no station of set $S_{k}$ other than $j$ transmits a packet longer than $l$, and $p$ (some $t x$ ) is the probability that, given that no station transmits a packet longer than $l$, at least some other station transmits. 
For the computation of $p$ (no $t x>l$ ), we index all the stations and refer with $S_{k, j}$ to the set of stations of $S_{k}$ with index smaller than $j$. Then, ${ }^{6}$

$$
p(\text { no } t x>l)=\prod_{m \in S_{k, j}}\left(1-\tau_{m} p\left(t_{m} \geq l\right)\right) \prod_{m \in S_{k} \backslash S_{k, j} \cup j}\left(1-\tau_{m} p\left(t_{m}>l\right)\right),
$$

where $p\left(t_{m}>l\right)$ and $p\left(t_{m} \geq l\right)$ are the probabilities that a transmission of station $m$ is longer than $l$ and longer than or equal to $l$, respectively.

$p($ some $t x)$ is computed as

$$
p(\text { some } t x)=1-\prod_{m \in S_{k, j}} \frac{1-\tau_{m}}{1-\tau_{m} p\left(t_{m} \geq l\right)} \prod_{m \in S_{k} \backslash S_{k, j} \cup j} \frac{1-\tau_{m}}{1-\tau_{m} p\left(t_{m}>l\right)} .
$$

Finally, by combining Eqs. (7)-(22) with Eq. (6), we can express the $\tau_{i}$ of a non saturated AC as a function of all the $\tau_{i}$ 's as follows:

$$
\tau_{i}^{\text {nonsat }}=\frac{\rho_{i}\left(1-p_{i}^{R+1}\right)\left(p(s) T_{s}+p(e) T_{e}+p(c) T_{\mathrm{c}}\right)}{l_{i}\left(1-\tau_{i}\right)^{n_{i}-1} \sum_{k=A_{i}}^{\Delta} p\left(A C_{k}\right) \prod_{j \in A C_{k} \backslash i}\left(1-\tau_{j}\right)^{n_{j}}},
$$

which terminates the analysis of a non saturated AC.

\subsection{Mixed Saturated and Non Saturated AC's Analysis}

We next combine the above analyses in order to obtain all the $\tau_{i}$ 's in the WLAN under stationary conditions. Then, we calculate the throughput and delay of each AC based on the obtained $\tau_{i}$ values.

From the above two subsections we have a method to compute the $\tau_{i}$ of a saturated and of a non saturated $\mathrm{AC}$, respectively; the remaining challenge lies in determining which AC's are saturated and which are not. For this, we proceed step by step as follows in order to classify all the AC's into two sets, one set with the saturated AC's and the other with the non saturated ones:

- In the first step, we consider that all AC's are saturated (i.e., they are all in the set of saturated AC's) and compute their saturation throughputs. Note that, from Section 3.1, we can express each $\tau_{i}$ of a saturated AC as a function of all the $\tau_{i}$ 's. Therefore, we have a system of $N$ nonlinear equations on the $\tau_{i}$ 's that can be resolved using numerical techniques. Once the $\tau_{i}$-values have been derived, we compute the throughput of all AC's by using Eqs. (7)-(22). ${ }^{7}$

- We next compare the throughputs resulting from the first step against the sending rates. If the throughput of an $\mathrm{AC}$ is larger than its sending rate, we consider from this step on that this AC is not saturated, and move it to the set of non saturated AC's. Indeed, such an AC cannot always have packets ready for transmission (and therefore cannot

\footnotetext{
6 The distinction in Eq. (21) between the stations of indexes smaller and larger than $j$ is done in order to avoid counting more than once the event when two or more stations transmit a packet of length $l$.

7 In Section 3.2, we calculated the $\tau_{i}$ of a non saturated AC by setting the throughput of the AC such that Eq. (6) is satisfied. Note that the part of that section where the throughput is computed as a function of all $\tau_{i}$ 's (Eqs. (7)-(22)) does not make any assumption about the status (saturated or not) of the AC, and therefore these equations can also be used to compute the throughput of a saturated $\mathrm{AC}$ as a function of all $\tau_{i}$ 's.
} 
be saturated), as otherwise it would be sending more packets than those generated by the station.

- In the second step, we take the new sets of saturated and non saturated AC's resulting from the first step and repeat the throughput computation. Note that, from Sections 3.1 and 3.2, we can express the $\tau_{i}$ of a saturated and of a non saturated AC, respectively, as a function of all the $\tau_{i}$ 's. Therefore, we have a new system of $N$ nonlinear equations from which we can obtain the $\tau_{i}$ 's and the corresponding throughputs.

- In the next step, we compare again the throughputs obtained in the previous step for the saturated AC's against their sending rates, and move those AC's whose throughput is larger than their sending rate to the set of non saturated AC's. ${ }^{8}$ After this reorganization of the sets, we repeat the throughput computation.

- The above is done iteratively until the resulting throughputs of all the saturated AC's are smaller than their sending rates. This last scenario represents a stable solution, and therefore the throughput values resulting from this step give us the throughput that each AC will obtain in the WLAN under stationary conditions.

Note that, as number of AC's $(N)$ is limited to 4 by the standard, the above process requires the execution of five steps at most (we start with all AC's saturated, at every step at least one $\mathrm{AC}$ becomes non saturated, and in the worst case we stop when all AC's are non saturated). In each step, a system of $N$ equations (i.e., no more than 4 ) has to be resolved. Therefore the computational cost of the proposed algorithm is reasonably low, as it requires solving no more than five systems of at most four equations. This is confirmed by the quantitative results on computational cost given in the following section.

The above terminates the analysis of the throughputs. For the computation of the delays, we use the delay model of [8], but taking the $\tau_{i}$-values obtained from the algorithm presented in this section, instead of the $\tau_{i}$ 's used in [8] which correspond to saturation conditions.

\section{Model Validation}

We validated the accuracy of the model by comparing the analytical values against those obtained via simulation. The simulations were performed for a WLAN with the system parameters of the IEEE 802.11b physical layer. We considered the following four AC's (which we name "voice," "video," "data," and "background," respectively):

- In the first $\mathrm{AC}, 80$ byte packets were generated every $10 \mathrm{~ms}$ (i.e., at a constant bit rate of $64 \mathrm{Kbps}$ ) to model the behavior of a $\mathrm{G} .711$ voice codec.

- In the second AC, we modeled video traffic with a variable bit rate source sending variable size packets with a constant interarrival time. The average bit rate of the source was set equal to $250 \mathrm{Kbps}$ and the packet length distribution was taken from the video traffic measurements of [15].

\footnotetext{
8 Note that an AC that was not saturated in the previous step can never become saturated again. In fact, if such an AC always had packets ready for transmission, it would obtain a throughput even larger than in the step where it became non saturated (since in the current step there are fewer saturated AC's). Therefore, it would be sending more packets than those generated.
} 


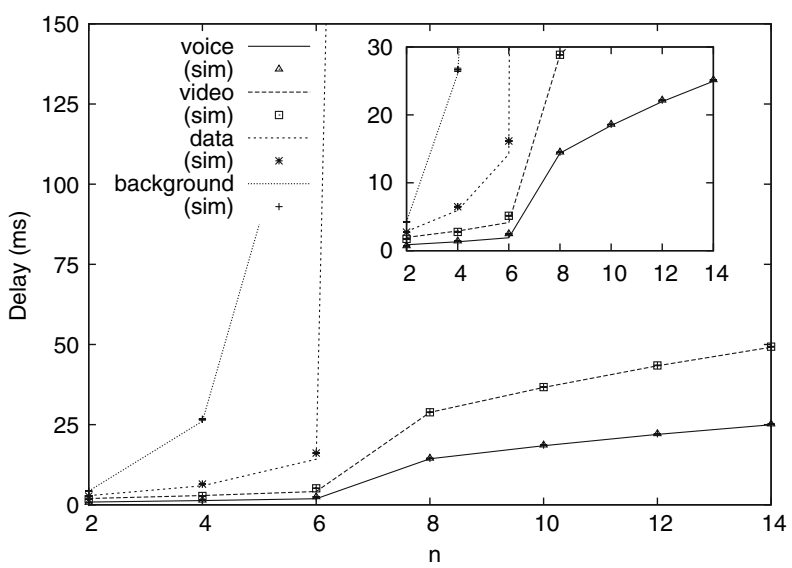

Figure 3. Average delay analysis validation.

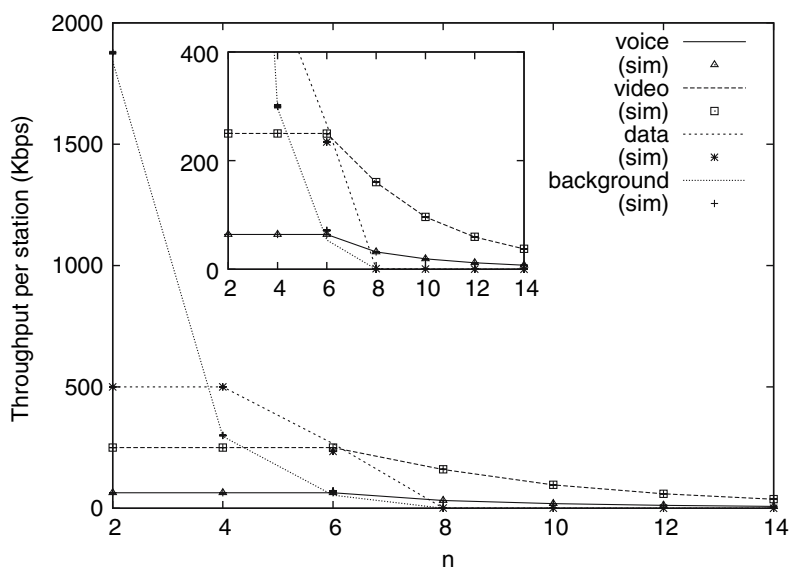

Figure 4. Throughput analysis validation.

- In the third AC, data traffic was generated according to a Poisson process of $500 \mathrm{Kbps}$ average bit rate and packet sizes following the distribution of the data traffic measurements of [16].

- Finally, in the fourth AC stations always had 1000 byte packets ready for transmission, modeling the behavior of a data transfer.

The configuration of each $\mathrm{AC}$ was derived from the recommendations given by the standard 802.11e [1]. Experiments were performed for a varying number of stations per AC (all AC's had $n$ stations each). The queue size of all the stations was set equal to 100 packets.

Figures 3 and 4 plot the average delay and throughput values obtained analytically (lines) and via simulation (points). Subplots are given for better observation of the low values. Simulation results are plotted with $95 \%$ confidence interval bars (note that confidence intervals are so small that they can barely be appreciated in the graphs).

From the figures, we observe that EDCA is effective in providing service differentiation. Both in terms of throughput and delay, higher priority AC's always perform better than lower priority ones. Furthermore, higher priority AC's also saturate later: AC 3 (data) saturates for $n>4$ while AC's 1 and 2 (voice and video) saturate for $n>6$ (AC 4 is by definition 
always saturated). Beyond this saturation point, in all AC's throughput decreases gradually with $n$, while delay increases drastically. For all cases, we have that analytical results match simulations remarkably well, which confirms the accuracy of our model.

We further validated the computational cost of the model by measuring the number of flops (floating point operations) required to execute a Matlab implementation of the algorithm. For all the presented experiments, results ranged from 25 to $325 \mathrm{Kflops}$. Although our implementation is not necessarily optimized, we believe that these results validate the model's computational efficiency. For instance, execution on a typical WLAN Access Point CPU with 100 MFlops capacity takes less than $10 \mathrm{~ms}$, which is fully acceptable for admission control.

\section{Conclusions}

In this paper, we have presented a model to analyze the behavior of the 802.11e EDCA protocol under non saturation conditions. Simulation results have shown that the model accurately captures the behavior of the protocol under $(i)$ typical source models (including voice, video, and data), (ii) realistic packet lengths as derived from measurements, and (iii) the configuration guidelines recommended by the standard. We conclude that, in contrast to previous works, our analysis can be used to model EDCA under realistic conditions.

The model presented here can be used for the design of admission control policies. Specifically, admission control can be implemented as follows: if (according to the model) the admittance of a new station in the WLAN degrades the service of the stations already present below a certain quality criterion, the new station is rejected. In line with the 802.11e standard [1], in a WLAN running under the infrastructure mode this algorithm can be executed at a centralized entity like, e.g., the Access Point.

\section{References}

1. IEEE 802.11e. Part 11: Wireless LAN Medium Access Control (MAC) and Physical Layer (PHY) specifications: Medium Access Control (MAC) Enhancements for Quality of Service (QoS). Supplement to IEEE 802.11 Standard, November 2005.

2. J. W. Robinson and T. S. Randhawa, "Saturation Throughput Analysis of IEEE 802.11e Enhanced Distributed Coordination Function", IEEE Journal on Selected Areas in Communications, Vol. 22, No. 5, pp. 917-928, 2004.

3. J. Hui and M. Devetsikiotis, "A unified model for the performance analysis of IEEE 802.11e EDCA", IEEE Transactions on Communications, Vol. 53, No. 9, pp. 1498-1510, 2005.

4. Z. Kong, D. H. K. Tsang, B. Bensaou, and D. Gao, "Performance Analysis of IEEE 802.11e Contention-Based Channel Access", IEEE Journal on Selected Areas in Communications, Vol. 22, No. 10, pp. 2095-2106, 2004.

5. V. Ramaiyan, A. Kumar, and E. Altman, "Fixed Point Analysis of Single Cell IEEE 802.11e WLANs: Uniqueness, Multistability and Throughput Differentiation", in Proceedings of ACM SIGMETRICS 2005, Banff, Alberta, Canada, 2005.

6. H. Zhu and I. Chlamtac, "Performance Analysis for IEEE 802.11e EDCF Service Differentiation", IEEE Transactions on Wireless Communications, Vol. 4, No. 4, pp. 1779-1788, 2005.

7. A. Banchs and L. Vollero, "Throughput Analysis and Optimal Configuration of 802.11e EDCA", Computer Networks, Vol. 50, No. 11, pp. 1749-1768, 2006.

8. A. Banchs and L. Vollero, "A Delay Model for 802.11e EDCA", IEEE Communications Letters, Vol. 9, No. 6, pp. 508-510, 2005. 
9. P. E. Engelstad and O. N. Osterbo, "Non-saturation and Saturation Analysis of IEEE 802.11e EDCA with starvation prediction", in Proceedings of the 8th ACM International Symposium on Modeling, Analysis and Simulation of Wireless and Mobile Systems (MSWIM'05), Montreal, Quebec, Canada, October 2005.

10. G.-R. Cantieni, Q. Ni, C. Barakat, and T. Turletti, "Performance Analysis of Finite Load Sources in 802.11b Multirate Environments", Computer Communications, Vol. 28, No. 10, 1095-1109, 2005.

11. J. W. Tantra, C. H. Foh, I. Tinnirello, and G. Bianchi, "Analysis of the IEEE 802.11e EDCA under Statistical Traffic”, in Proceedings of ICC'06, Istanbul, Turkey, 2006.

12. K. Duffy, D. Malone, and D. Leith, "Modeling the 802.11 Distributed Coordination Function in Non-Saturated Conditions", IEEE Communications Letters, Vol. 9, No. 8, pp. 715-717, 2005.

13. B. Li and R. Battiti, "Analysis of the 802.11 DCF with Service Differentiation Support in Non-Saturation Conditions", in Proceedings of the Fifth International Workshop on Quality of Future Internet Services (QofIS'05), Barcelona, Catalonia, Spain, 2004.

14. H. Wu, Y. Peng, K. Long, S. Cheng, and J. Ma, "Performance of Reliable Transport Protocol Over IEEE 802.11 Wireles LAN: Analysis and Enhancement", in Proceedings of IEEE INFOCOM'O2, New York, NY, USA, 2002.

15. L. Muscariello, M. Mellia, M. Meo, R. Lo Cigno, and M. Ajmone, “A Simple Markovian Approach to Model Internet Traffic at Edge Routers", COST279, Technical Document, No. TD(03)032, 2003.

16. K. Claffy, G. Miller, and K. Thompson, "The Nature of the Beast: Recent Traffic Measurements from an Internet Backbone", in Proceedings of INET'98, Geneve, Switzerland, 1998.

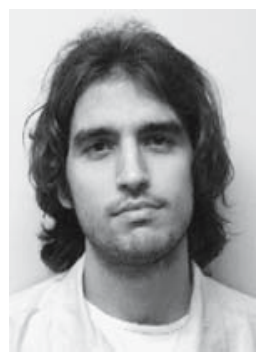

Pablo Serrano was born in Tarifa, Spain, on May 17, 1979. He received a M.Sc. degree in Telecommunications from the University Carlos III of Madrid in 2002. Since that date he is a Ph.D. candidate and a lecturer at the Telematics Department of the same university. His current research interests are performance evaluation and resource allocation of WLAN networks.

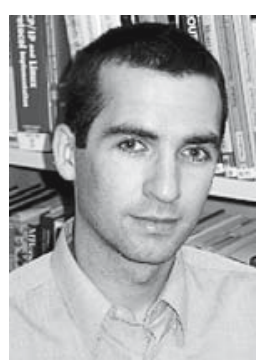

Albert Banchs received his M.Sc. and Ph.D. degrees in Telecommunications from the Technical University of Catalonia in 1997 and 2002, respectively. His Ph.D. received the national award for best thesis on Broadband Networks granted by the Professional Association of Telecommunication Engineers. He worked for the International Computer Science Institute, Berkeley, in 1997, for Telefonica I+D, Madrid, in 1998 and for NEC Network Laboratories, Heidelberg, from 1998 to 2003. Since 2003 he is with the University Carlos III of Madrid. 
Dr. Banchs has published over 30 articles in international conferences and journals. He is an Associate Editor of the IEEE Communications Letters and has been member of the Technical Program Committee of several conferences and workshops, including ICC, GLOBECOM and INFOCOM. His current research interests include resource allocation, QoS and performance evaluation of wireless and wired networks.

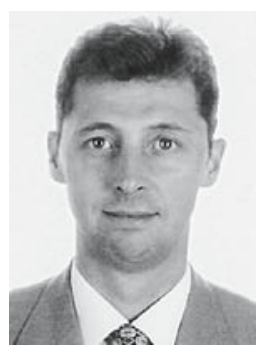

Arturo Azcorra received a M.Sc. degree in Telecommunications from the Technical University of Madrid in 1986, and a Ph.D. degree from the same university in 1989. His Ph.D. received the national award for best thesis, jointly granted by the Professional Association of Telecommunication Engineers and the National Association of Electronic Industries. In 1993 he obtained an MBA from Instituto de Empresa, Madrid. He was a lecturer at the Technical University of Madrid from 1987 to 1990, when he was promoted to associate professor. In 1998 he joined the University Carlos III of Madrid, where he has stayed up to now as full professor. Since 1998 he is also deputy Vice-Provost for Academic Infrastructures at the University Carlos III of Madrid. Prof. Azcorra has been program committee member of several editions of IEEE-PROMS, IDMS, QofIS, CONEXT and IEEEINFOCOM. He is general cochair for CONEXT'05 and TPC co-chair for INFOCOM'06. His list of papers published in national and international journals, books and conferences is over 100 titles. Current research projects include broadband networks, multicast teleservices, active networks and advanced IP networks. 